\title{
Long observations of the BALQSO LBQS 2212-1759 with XMM-Newton ${ }^{\star}$
}

\author{
J. Clavel, ${ }^{1}$ N. Schartel ${ }^{2}$, and L. Tomas ${ }^{2}$ \\ 1 Research \& Scientific Support Department, ESTEC, SCI-SA, Postbus 2992200 AG - Noordwijk, The Netherlands \\ e-mail: Jean.Clavel@esa.int \\ 2 XMM-Newton Science Operations Centre, ESAC, Apartado 50727, 28080 Madrid, Spain \\ e-mail: [nscharte; ltomas]@xmm.vilspa.esa.es
}

Received 6 September 2004 / Accepted 9 September 2005

\section{ABSTRACT}

Very long (172 ks effective exposure time) observations of the BALQSO LBQS 2212-1759 with XMM-Newton yield a stringent upper-limit on its $0.2-10 \mathrm{keV}$ (rest- frame $0.64-32.2 \mathrm{keV}$ ) flux, $F_{0.64-32.2} \leq 6 \times 10^{-17} \mathrm{erg} \mathrm{cm}^{-2} \mathrm{~s}^{-1}$, while simultaneous UV and optical observations reveal a rather blue spectrum extending to $650 \AA$ in the source rest frame. These results are used to set a tight upper-limit on its optical to X-ray spectral index $\alpha_{\mathrm{ox}} \leq-2.56$. Given the HI-BAL nature of LBQS 212-1759, its X-ray weakness is most likely due to intrinsic absorption. If this is the case, and assuming that the intrinsic $\alpha_{\mathrm{ox}}$ of LBQS 2212-1759 is -1.63 - a value appropriate for a radio-quiet quasar of this luminosity - one can set a lower limit on the X-ray absorbing column $N_{\mathrm{H}} \geq 3.4 \times 10^{25} \mathrm{~cm}^{-2}$. Such a large column has a Thomson optical depth to electron scattering $\tau_{\mathrm{Th}} \geq 23$, sufficient to extinguish the optical and UV emission. The contradiction becomes even more acute if the gas is neutral since the opacity in the Lyman continuum becomes extremely large, $\tau_{\mathrm{Ly}} \geq 2 \times 10^{8}$, conflicting with the source detection below $912 \AA$. This apparent contradiction probably means that our lines-of-sight to the X-ray and to the UV emitting regions are different, such that the gas completely covers the compact X-ray source but only partially the more extended source of ultraviolet photons. An extended $\left(\simeq 1^{\prime}\right) X$-ray source is detected $\sim 2^{\prime}$ to the south-east of the QSO. Given its thermal spectrum and temperature $(1.5 \leq T \leq 3.0 \mathrm{keV})$, it is probably a foreground $(0.29 \leq z \leq 0.46)$ cluster of galaxies.

Key words. quasars: absorption lines - quasars: individual: LBQS 2212-1759 - X-rays: individuals: LBQS 2212-1759 - galaxies: active $\mathrm{X}$-rays: galaxies - ultraviolet: galaxies - galaxies: active

\section{Introduction}

Broad Absorption Line (BAL) quasars are characterized by broad and blueshifted absorption troughs in their spectrum, from resonance transitions such as $\operatorname{CIV} \lambda 1550$, Ly $\alpha \lambda 1216$, $\mathrm{NV} \lambda 1240$, indicating the presence of a high velocity (up to $50000 \mathrm{~km} \mathrm{~s}^{-1}$ ) outflow along the line-of-sight (LOS) to the nucleus. Taking into account selection biases, BALQSOs represent $22 \pm 4 \%$ of the radio-quiet quasar population (Hewett \& Foltz 2003). The fraction of BALQSOs that are radio-loud is approximately the same as that of non-BAL quasars, but there appears to be a deficit of broad absorption line objects at large radio luminosities (Menou et al. 2001; Becker et al. 2000). Because of the overall similarity of their continuum and emission line properties with those of non-absorbed quasars, it is sometime thought that BALQSOs are "normal" quasars seen at a specific viewing angle such that our LOS intercepts a nuclear wind (Weyman et al. 1991). The wind possibly originates from the accretion disk and is driven out radially by radiation

^ This work is based on observations obtained with XMM-Newton, an ESA science mission with instruments and contributions directly funded by ESA Member States and the USA (NASA). pressure (Murray et al. 1995). A hydrodynamic model for the BAL wind was developed by Proga et al. (2000). In the empirical scenario proposed by Elvis (2000), the wind arises vertically from a narrow range of disk radii and bends outward to a cone angle of $60^{\circ}$ with a divergence angle of $6^{\circ}$. In this type of models, it is the solid angle covered by the outflow that determines the fraction of BAL quasars. An alternative class of models speculates that the BAL phenomenon represents an early "cocoon" phase in the evolution of a QSO (e.g. Briggs et al. 1984). Although his results are based on a small sample that contains only 4 BALQSOs, Boroson (2002) lent some credibility to this idea by showing that BALQSOs occupy a specific location in the quasar parameter space, characterized by large accretion rates and luminosities, close to the Eddington limit. The evolution scenario is also supported by the large fraction of BALQSOs found in a spectroscopic follow-up to the VLA FIRST survey - 29 radio-selected BALQSOs (Becker et al. 2000) - since the properties of the sample appear inconsistent with simple unified models.

BALQSOs are invariably X-ray weak or silent (Green et al. 1995; Green \& Mathur 1996; Gallagher et al. 1999), suggesting the presence of very large absorbing columns, $N_{\mathrm{H}} \geq 10^{23} \mathrm{~cm}^{-2}$, 
2-3 orders of magnitudes larger than those inferred from UV absorption line studies. This discrepancy led to the conclusion that the bulk of the absorbing gas is highly ionised and thus mostly transparent in the ultraviolet while still providing large X-ray opacities. However, it was subsequently realized that the column densities derived from curve of growth analysis of absorption lines may be severely underestimated. High resolution and high signal-to-noise ratio UV spectra show that the lines are saturated despite the existence of residual flux at their bottom (e.g. Arav et al. 1999; Wang et al. 1999). The residual flux may be due to partial covering of the continuum source or to the scattering of part of its emission back into our LOS, as indicated by the higher degree of polarisation of BALQSOs as compared to non-BAL quasars (e.g. Schmidt \& Hines 1999; Ogle et al. 1999).

Here we present very sensitive observations of the $z=$ 2.217 BALQSO LBQS 2212-1759 (Morris et al. 1991) performed with XMM-Newton. This quasar was selected because of its optical brightness $\left(m_{B}=17.94\right)$ and tentative 3 -sigma detection in the soft-X-ray band with ROSAT (Green et al.1995). LBQS 2212-1759 displays two CIV $\lambda 1548$ absorption troughs blue-shifted respectively by $\simeq-6300$ and $-4000 \mathrm{~km} \mathrm{~s}^{-1}$ with respect to its systemic velocity (Korista et al. 1993).

\section{Observations and data reduction}

LBQS 2212-1759 was observed twice with XMM-Newton (Jansen et al. 2001). The first observation took place from $22 \mathrm{~h} 15 \mathrm{~m}$ (UT) on November 17, 2000 to $17 \mathrm{~h} 44 \mathrm{~m}$ on November 18 , under conditions of extremely low particle radiation background. The quasar was observed again one year later, from $22 \mathrm{~h} 17 \mathrm{~m}$ on November 17, 2001 to $04 \mathrm{~h} 53 \mathrm{~m}$ on November 19. On each occasion, data were collected simultaneously with all X-ray instruments on board XMM-Newton: the EPIC-pn (Strüder et al. 2001), the two EPIC-MOS (Turner et al.2001), as well as the two $R G S$ spectrographs (den Herder et al. 2001). As expected, the last did not yield any useful information and the corresponding data are not discussed further in the remainder of this paper. A log of the X-ray observations is provided in Table 1, where we give the XMM-Newton observation identifier in Col. 1, the start date of the observation in Col. 2 and the exposure times with the three EPIC pn and EPIC MOS instruments in Cols. 3 and 4, respectively. Note the very long cumulative exposure time of the X-ray observations. In the case of the most sensitive EPIC-pn instrument, it reaches 221352 s. Even after data screening the cumulative useful integration time of the EPIC pn data remains $172628 \mathrm{~s}$.

The X-ray $p n$ and $M O S$ data were reduced and analyzed in a standard fashion using the SAS v5.3. The pipe-line products of observation 0106660601 provide 44 positional coincidences between sources of the USNO-A2.0 Catalogue (Monet et al. 1998) and X-ray sources in the field of LBQS 2212-1759. Of these 44 coincidences, $35 \mathrm{X}$-ray sources have only one, three $\mathrm{X}$-ray sources have two and one X-ray source has three optical counterparts. Restricting to the $35 \mathrm{X}$-ray sources with a unique optical identification, we infer a mean offset of $2.2^{\prime \prime}$ between the X-ray position and the optical source coordinates.
Table 1. Details of the XMM-Newton X-ray observations.

\begin{tabular}{cccc}
\hline \hline Obs ID & Start Date & \multicolumn{2}{c}{ exposure times (s) } \\
& & EPIC pn & EPIC MOS 1 and 2 \\
\hline 0106660101 & $2000-11-17$ & 55718 & $2 \times 57824$ \\
0106660201 & $2000-11-18$ & 50618 & $2 \times 52724$ \\
0106660401 & $2001-11-18$ & 0 & $2 \times 33050$ \\
0106660501 & $2001-11-17$ & 8208 & $2 \times 10822$ \\
0106660601 & $2001-11-17$ & 106808 & $2 \times 109422$ \\
\hline
\end{tabular}

Table 2. $3-\sigma$ Upper limits to the X-ray flux of LBQS 2212-1759 derived from the EPIC-pn observations.

\begin{tabular}{ccc}
\hline $\begin{array}{c}\text { energy band } \\
(\mathrm{keV})\end{array}$ & $\begin{array}{c}\text { Count rate } \\
\left(10^{-5} \mathrm{~s}^{-1}\right)\end{array}$ & $\begin{array}{c}\text { Flux } \\
\left(10^{-16} \mathrm{erg} \mathrm{cm}^{-2} \mathrm{~s}^{-1}\right)\end{array}$ \\
\hline $0.2-0.5$ & $\leq 9.03$ & $\leq 1.69$ \\
$0.5-2.0$ & $\leq 9.25$ & $\leq 2.74$ \\
$2.0-10.0$ & $\leq 8.65$ & $\leq 14.2$ \\
$5.0-10.0$ & $\leq 6.16$ & $\leq 20.1$ \\
$0.2-10.0$ & $\leq 1.48$ & $\leq 0.64$ \\
\hline
\end{tabular}

Note that out of these $35 \mathrm{X}$-ray sources with a unique identification, 25 (i.e. $63 \%$ ) lie within $2^{\prime \prime}$ of their optical counterpart. The BAL quasar was not detected in either of the X-ray instruments. The nearest detected X-ray point source is $\sim 0.5^{\prime}$ away from the nominal position of LBQS 2212-1759 (RA = 22:15:31.6; Dec = 17:44:06 - J2000)

From the rms background count fluctuations in a $9 \times 9$-pixel cell centered on the expected source position, we computed $3 \sigma$ upper limits to the count rate in various energy bands. The HEW of the EPIC-pn point-spread function is $14^{\prime \prime}$ and one CCD pixel projects onto an area of $4.1 \times 4.1^{\prime \prime}$ on the sky. From the XMM-Newton observation of Q 0056-363, we determined the ratios between the total count rate of a faint point-source and the count rates measured in a $9 \times 9$-pixel cell centered on the source for each of the energy bands. These ratio were then applied to the cell upper limits to derive effective upper limits to the source count rate. The results are listed in Table 2. These count rates were converted into flux upper limits using the PIMMS software available on-line at the HEASARC website. The results are given in Table 2 were we only list the results from the EPIC pn data, since the less sensitive EPIC MOS detectors yield consistent but significantly higher and therefore less constraining limits.

In an attempt to understand why LBQS 2212-1759 was marginally detected by Green et al. (1995), we checked their original ROSAT image. There are definitely no excess counts at the centre of their $3^{\prime}$ radius extraction circle, clearly ruling out the presence of a point-source. However, the merged EPIC data reveal the existence of a weak extended source, centered at $\mathrm{RA}=22: 15: 37$ and $\mathrm{Dec}=-17: 45: 35$ and whose radius is $1.0^{\prime}$. This source is most probably a foreground cluster of galaxies since its spectrum is well described by a Mekal spectrum with temperature in the range $1.5-3 \mathrm{keV}$ and a redshift between 0.29 and 0.46 . Whatever its origin, this source clearly lies within the ROSAT extraction region and is likely the origin of the false 
detection of LBQS 2212-1759 by Green et al. (1995). The extended source was too weak to appear in the ROSAT All Sky Survey catalogue (1RXS) and could not therefore be taken into account by Green et al. (1995). Note that there are no EPIC point-sources within the 10-20' annulus that these authors used to measure the background in the ROSAT image.

In parallel to the X-ray observations, a series of optical and ultraviolet broad-band filter images of the QSO field were obtained with the Optical Monitor telescope (OM; Mason et al. 2001) on board XMM-Newton. The $O M$ data were reprocessed with the SAS version 6.0 using the script omichain. For each broad-band filter image, the corrected net count rate of the QSO was read-off directly from the SWSRLI output files, which lists all sources automatically detected by the SAS software, together with their count rate, statistical significance, measured coordinates, the associated errors and various data quality indicators. We used the close-by 13th mag star $S 3211320188$ from the HST guide-star catalog to correct for small $\left(\leq 4^{\prime \prime}\right)$ residual astrometric distortions in the $O M$ coordinate system. After correction, the QSO coordinates as measured with $O M$ agree to better than $1^{\prime \prime}$ with $N E D$ catalog coordinates. The count rates were converted into fluxes following the recipe provided on the SAS web page at URL xmm.vilspa.esa.es/ sas/documentation/watchout/uvflux.html. The final fluxes are listed in Table 3, which provides: the observation identifier in Col. 1, the OM exposure number in Col. 2, the date and U.T. time of the start of the exposure expressed as a fractional day of 2000 in Col. 3, the filter identifier in Col. 4 and the flux with its associated statistical rms error in Col. 5. Early observations with the less sensitive UVW2 filter had exposure times that were too short and did not yield statistically significant detections. In such cases, $3-\sigma$ upper limits are listed in Table 3.

A $\chi^{2}$ test shows that the flux of LBQS 2212-1759 remained constant within the measurement uncertainties in all 6 filters. The reduced chi-squares (d.o.f.) corresponding to the hypothesis of a constant flux are $\chi_{v}^{2}=0.88(8), 0.53(9), 1.19$ (9), 0.52 (10) and 1.20 (6) for the $V, B, U, U V W 1$ and $U V M 2$ filters, respectively. We therefore averaged the results from individual exposures and computed the weighted mean flux in each filter and the error on the mean. The near-IR $J, H$ and $K$ fluxes of LBQS 2212-1759 were retrieved from the 2MASS catalog (Kleinmann 1994; Barkhouse \& Hall 2001). All fluxes were finally corrected for foreground galactic extinction $\left(E_{b-v}=0.026\right.$; Schlegel et al. 1998; Cardelli et al. 1989). The results are given in Table 4, where we list the origin of the data in Col. 1, the effective wavelength and band-pass of the filter in the observer's frame in Cols. 2 and 3, respectively, the effective wavelength and band-pass of the filter in the quasar rest-frame in Cols. 4 and 5, respectively and the averaged dereddened flux in Col. 6.

The rest-frame optical to EUV energy distribution of LBQS 2212-1759, is shown in Fig. 1. A 1992 spectrum of LBQS 2212-1759 (Korista et al.1993) is also displayed for comparison. Note that while the flux increased by $46 \%$ in the $\mathrm{OM}-\mathrm{V}$ band during the 8.5 years interval between the two observations, the spectral shape remained very similar.

\section{Implications and discussion}

The UV-optical SED of LBQS 2212-1759 is typical of a high redshift QSO; at wavelengths longer than that of H-Ly $\alpha \lambda 1215$, it displays a blue spectrum giving rise to an excess emission, the so-called "big blue bump", usually attributed to the thermal emission of an accretion disk. A power-law $\left(F_{v} \propto v^{\alpha}\right)$ fit to the 5 data-points with $\lambda_{\text {rest }} \geq 1215 \AA$ yields a spectral in$\operatorname{dex} \alpha=-0.96 \pm 0.18$. Such an index is within the range of optical-UV slopes observed in the general quasar population. As noted by several authors (see e.g. Elvis et al. 1994), the spread in indices is fairly large. In the Francis et al. (1991) sample of 688 LBQS quasars for instance, indices vary from -1.5 to +1 . The $1050-2200 \AA$ spectral index of the average composite quasar of Zheng et al. (1997) is $-0.86 \pm 0.01$, while the mean 1285-5100 ̊ spectral index of the radio-quiet QSO sample of Kuhn et al. (2001) is $-0.32 \pm 0.28(1-\sigma)$ and independent of redshift. The composite quasar spectrum of Vanden Berk et al. (2001), obtained by averaging 2200 QSO spectra from the Sloan Digital Sky Survey, has a 1350-4230 A index of -0.44 . At wavelengths shorter than $1215 \AA$, the LBQS 2212-1759 spectrum steepens to a softer spectral index $\alpha=-2.62 \pm 0.16$. Again, such a steepening is not unusual amongst quasars and has been reported by several authors (e.g. Zheng et al. 1997; Kuhn et al. 2001; Vanden Berk et al. 2001). In a "normal" high redshift quasar, this sharp steepening of the spectrum in the EUV is due to the onset of many intervening Lyman series absorption systems along the line-of-sight, the so-called "Lyman alpha forest". In a BALQSO like LBQS 2212-1759, the steepening can also be attributed, at least partly, to resonance absorption within the BAL nuclear outflow.

The ultraviolet spectrum of LBQS 2112-1759 is however difficult to reconcile with the above stringent upper limits on its X-ray flux. The optical-to-X-ray spectral index of a quasar (Zamorani et al. 1981), $\alpha_{\mathrm{ox}}$, is defined as the spectral index of an hypothetical power-law connecting its flux density at $2500 \AA$ and $2.0 \mathrm{keV}$ in the QSO rest-frame, $\alpha_{\mathrm{ox}}=0.384 \log \frac{F_{2 \mathrm{keV}}}{F_{2500}}$. In radio-quiet non-BAL quasars, it is observationally confined to a range $-1.1 \geq \alpha_{\mathrm{ox}} \geq-1.9$ with a weak dependence on the source luminosity (Vignali et al. 2003; Strateva et al. 2005). Using the same cosmological parameters as these authors, the monochromatic luminosity of LBQS 2112-1759 at $2500 \AA$ (rest wavelength) is $L_{2500}=1.7 \times 10^{31} \mathrm{erg} \mathrm{s}^{-1} \mathrm{~Hz}^{-1}$, which, according to Eq. (6) of Strateva et al., predicts $\alpha_{\mathrm{ox}}=-1.63 \pm 0.03$. Assuming a canonical photon spectral index $\Gamma=-1.9$ for the 0.2-10 keV spectrum of LBQS 2212-11759 (e.g. Laor et al. 1997), and using the X-ray flux upper limits of Table 2, one can infer an upper limit to the monochromatic flux density at a rest energy of $2 \mathrm{keV}\left(E_{\mathrm{obs}}=0.621 \mathrm{keV}\right), F_{v}(2 \mathrm{keV}) \leq$ $1.0 \times 10^{-8} \mathrm{mJy}$. One can derive the flux at $\lambda_{\text {rest }}=2500 \AA$ by interpolation between the de-reddened fluxes in the $\mathrm{J}$ and $\mathrm{V}$ bands. Combining the two yields $\alpha_{\text {ox }} \leq-2.56$, steeper by $\sim 1$ dex than the index predicted for a radio-quiet quasar of the same luminosity as LBQS 2212-1759. This is illustrated in Fig. 2, where we plot the overall Spectral Energy Distribution (SED) of LBQS 2212-1759. The upper limit to its $2 \mathrm{keV}$ flux is 263 times lower than that predicted by extrapolation of its $2500 \AA$ flux density with a power-law of index $\alpha_{\mathrm{ox}}=-1.63$. 
Table 3. Optical and UV fluxes measured though the various OM filters.

\begin{tabular}{|c|c|c|c|c|}
\hline Obs ID & Exposure \# & $\begin{array}{c}\text { Start date } \\
(2000 \text { day \#) }\end{array}$ & Filter & $\begin{array}{c}\text { Flux } \\
\left(10^{-16} \mathrm{erg} \mathrm{cm}^{-2} \mathrm{~s}^{-1} \AA^{-1}\right)\end{array}$ \\
\hline 106660101 & 10 & 321.78944 & $\bar{V}$ & $2.79 \pm 0.33$ \\
\hline 106660101 & 401 & 321.80242 & V & $3.32 \pm 0.33$ \\
\hline 106660101 & 402 & 321.81542 & $V$ & $2.72 \pm 0.33$ \\
\hline 106660101 & 403 & 321.82841 & $V$ & $2.80 \pm 0.32$ \\
\hline 106660101 & 404 & 321.84140 & $V$ & $3.00 \pm 0.33$ \\
\hline 106660201 & 6 & 322.54738 & V & $2.77 \pm 0.30$ \\
\hline 106660201 & 401 & 322.56269 & $V$ & $2.42 \pm 0.30$ \\
\hline 106660201 & 402 & 322.57797 & V & $2.41 \pm 0.30$ \\
\hline 106660201 & 403 & 322.59329 & $V$ & $2.47 \pm 0.29$ \\
\hline 106660101 & 8 & 321.91927 & $B$ & $4.33 \pm 0.22$ \\
\hline 106660101 & 409 & 321.93226 & $B$ & $4.36 \pm 0.22$ \\
\hline 106660101 & 410 & 321.94523 & $B$ & $4.42 \pm 0.22$ \\
\hline 106660101 & 411 & 321.95822 & $B$ & $4.25 \pm 0.21$ \\
\hline 106660101 & 412 & 321.97122 & $B$ & $3.95 \pm 0.22$ \\
\hline 106660201 & 9 & 322.70034 & $B$ & $4.23 \pm 0.20$ \\
\hline 106660201 & 409 & 322.71564 & $B$ & $4.17 \pm 0.20$ \\
\hline 106660201 & 410 & 322.73094 & $B$ & $4.12 \pm 0.20$ \\
\hline 106660201 & 411 & 322.74624 & B & $4.35 \pm 0.20$ \\
\hline 106660201 & 412 & 322.76154 & $B$ & $4.02 \pm 0.20$ \\
\hline 106660101 & 7 & 321.85435 & $U$ & $3.84 \pm 0.20$ \\
\hline 106660101 & 405 & 321.86733 & $U$ & $4.13 \pm 0.20$ \\
\hline 106660101 & 406 & 321.88032 & $U$ & $4.12 \pm 0.20$ \\
\hline 106660101 & 407 & 321.89332 & $U$ & $4.11 \pm 0.20$ \\
\hline 106660101 & 408 & 321.90630 & $U$ & $3.59 \pm 0.20$ \\
\hline 106660201 & 8 & 322.62387 & $U$ & $4.00 \pm 0.18$ \\
\hline 106660201 & 405 & 322.63916 & $U$ & $3.83 \pm 0.18$ \\
\hline 106660201 & 406 & 322.65446 & $U$ & $3.84 \pm 0.18$ \\
\hline 106660201 & 407 & 322.66976 & $U$ & $4.24 \pm 0.18$ \\
\hline 106660201 & 408 & 322.68506 & $U$ & $3.73 \pm 0.18$ \\
\hline 106660101 & 9 & 321.98869 & $U V W 1$ & $3.85 \pm 0.20$ \\
\hline 106660101 & 413 & 322.01070 & $U V W 1$ & $3.70 \pm 0.20$ \\
\hline 106660101 & 414 & 322.05354 & $U V W 1$ & $3.60 \pm 0.20$ \\
\hline 106660101 & 415 & 322.07557 & $U V W 1$ & $3.57 \pm 0.19$ \\
\hline 106660101 & 416 & 322.09757 & $U V W 1$ & $3.73 \pm 0.20$ \\
\hline 106660201 & 10 & 322.78260 & $U V W 1$ & $3.65 \pm 0.18$ \\
\hline 106660201 & 413 & 322.80948 & $U V W 1$ & $3.68 \pm 0.18$ \\
\hline 106660201 & 414 & 322.83636 & $U V W 1$ & $3.59 \pm 0.18$ \\
\hline 106660201 & 415 & 322.86323 & $U V W 1$ & $3.60 \pm 0.18$ \\
\hline 106660201 & 416 & 322.89011 & $U V W 1$ & $3.68 \pm 0.18$ \\
\hline 106660501 & 6 & 686.10035 & $U V W 1$ & $3.39 \pm 0.17$ \\
\hline 106660601 & 11 & 687.44819 & $U V W 1$ & $3.48 \pm 0.11$ \\
\hline 106660101 & 10 & 322.11957 & $U V M 2$ & $3.28 \pm 0.39$ \\
\hline 106660101 & 418 & 322.16359 & $U V M 2$ & $2.78 \pm 0.39$ \\
\hline 106660101 & 419 & 322.18561 & $U V M 2$ & $2.97 \pm 0.39$ \\
\hline 106660101 & 420 & 322.20762 & $U V M 2$ & $2.99 \pm 0.39$ \\
\hline 106660401 & 9 & 685.93929 & $U V M 2$ & $2.41 \pm 0.26$ \\
\hline 106660601 & 13 & 687.65182 & $U V M 2$ & $2.50 \pm 0.24$ \\
\hline 106660101 & 11 & 322.23667 & $U V W 2$ & $<6.60$ \\
\hline 106660101 & 422 & 322.30894 & $U V W 2$ & $<7.19$ \\
\hline 106660101 & 423 & 322.34508 & $U V W 2$ & $<6.56$ \\
\hline 106660101 & 424 & 322.38120 & $U V W 2$ & $3.48 \pm 0.70$ \\
\hline 106660401 & 10 & 686.02503 & $U V W 2$ & $1.33 \pm 0.58$ \\
\hline 106660501 & 8 & 686.15948 & $U V W 2$ & $<8.16$ \\
\hline 106660601 & 14 & 687.74334 & $U V W 2$ & $2.20 \pm 0.58$ \\
\hline 106660601 & 15 & 687.83490 & $U V W 2$ & $2.15 \pm 0.58$ \\
\hline
\end{tabular}




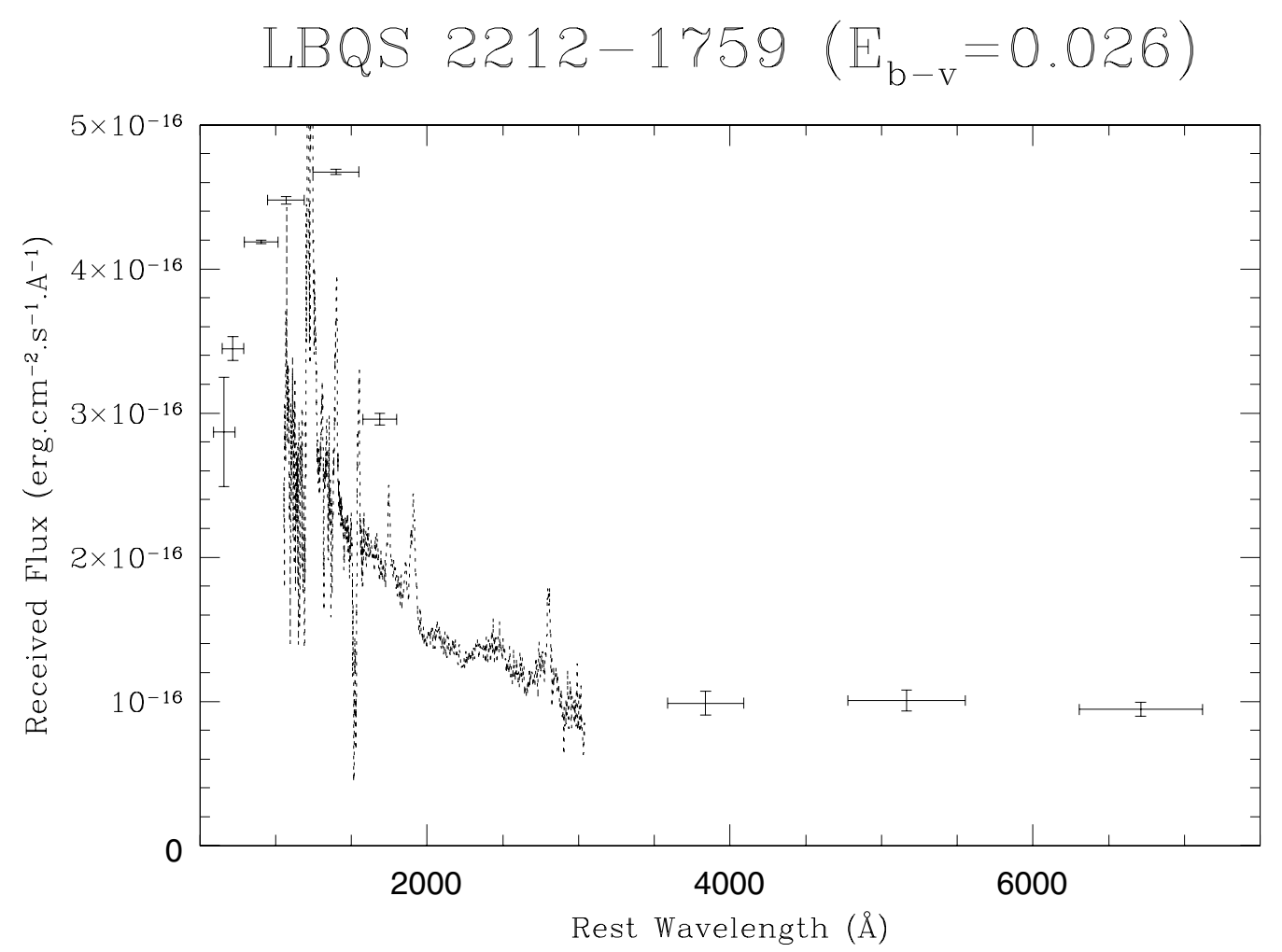

Fig. 1. The flux distribution of LBQS 2212-1759 from $6711 \AA$ to $659 \AA$ (rest wavelengths). All data-points are from the present study, except for the 3 longest wavelengths ones which were retrieved from the 2MASS catalogue. The 1992 spectrum from Korista (1993) is also shown for comparison. All flux values are in the observer's frame and have been corrected for foreground galactic extinction $\left(E_{b-v}=0.026\right)$.

Table 4. The flux of LBQS 2212-1759 as a function of wavelength, from the near-IR to the EUV. The values in the optical and UV have been obtained by averaging fluxes from individual OM exposures. All fluxes have been corrected for foreground galactic reddening.

\begin{tabular}{|c|c|c|c|c|c|}
\hline Instrument & $\lambda_{\mathrm{obs}}$ & $\begin{array}{r}\Delta \lambda_{\mathrm{obs}} \\
(\AA)\end{array}$ & $\lambda_{\text {rest }}$ & $\Delta \lambda_{\text {rest }}$ & $\begin{array}{c}\text { Flux } \\
\left(10^{-16} \mathrm{erg} \mathrm{cm}^{-2} \mathrm{~s}^{-1} \AA^{-1}\right)\end{array}$ \\
\hline 2MASS $K$ & 21590 & 2620 & 6711 & 814 & $0.945 \pm 0.047$ \\
\hline 2MASS $H$ & 16620 & 2510 & 5166 & 780 & $1.006 \pm 0.071$ \\
\hline 2MASS $J$ & 12350 & 1620 & 3839 & 504 & $0.987 \pm 0.082$ \\
\hline OM- $V$ & 5430 & 710 & 1688 & 221 & $2.957 \pm 0.040$ \\
\hline OM- $B$ & 4500 & 980 & 1399 & 305 & $4.673 \pm 0.018$ \\
\hline OM-U & 3440 & 780 & 1069 & 242 & $4.478 \pm 0.026$ \\
\hline $\mathrm{OM}-U V W 1$ & 2910 & 710 & 905 & 221 & $4.186 \pm 0.013$ \\
\hline OM-UVM2 & 2310 & 460 & 718 & 143 & $3.447 \pm 0.082$ \\
\hline OM- $U V W 2$ & 2120 & 460 & 659 & 143 & $2.868 \pm 0.379$ \\
\hline
\end{tabular}

Assuming that the difference is entirely due to intrinsic absorption of the X-ray flux, one can infer a lower limit to the required absorbing column, $N_{\mathrm{H}} \geq 3.4 \times 10^{25} \mathrm{~cm}^{-2}$. Note that this result depends only weakly on the value assumed for the X-ray spectral index. For instance, using $\Gamma=-2.5$ instead of -1.9 hardly changes the results to $N_{\mathrm{H}} \geq 2.6 \times 10^{25} \mathrm{~cm}^{-2}$.

If the gas was neutral, an absorbing column as large as or larger than $3.4 \times 10^{25} \mathrm{~cm}^{-2}$ would create an optical depth at the Hydrogen Lyman limit, $\tau_{\text {Ly }} \geq 2.2 \times 10^{8}$, more than sufficient to extinguish all radiation at wavelengths shorter than $912 \AA$. This is not the case however, since LBQS 2212-1759 is detected to $\lambda_{\text {rest }}=659 \AA$. Hence, the X-ray absorbing gas cannot be neutral and cover the UV continuum source. However, even if the gas is fully ionised, the Thomson optical depth to electron scattering corresponding to the above column, $\tau_{\text {th }} \geq 23$, is sufficient to attenuate the flux by a factor $\simeq 10^{10}$ and make LBQS 2212-1759 invisible at all wavelengths except in the $\gamma$ ray regime. Another difficulty is that, unless the gas is completely free of dust, extinction will wipe-out any emerging UV and optical photon. Even if the dust to gas ratio is 100 times lower than the average galactic value (e.g. Gorenstein 1975), an absorbing column of $3.4 \times 10^{25} \mathrm{~cm}^{-2}$ would still generate $\sim 150$ mag of visual extinction and approximately ten times more in the far ultraviolet.

We are thus left with an inconsistency: on the one hand, LBQS 2212-1759 is detected with high statistical significance 


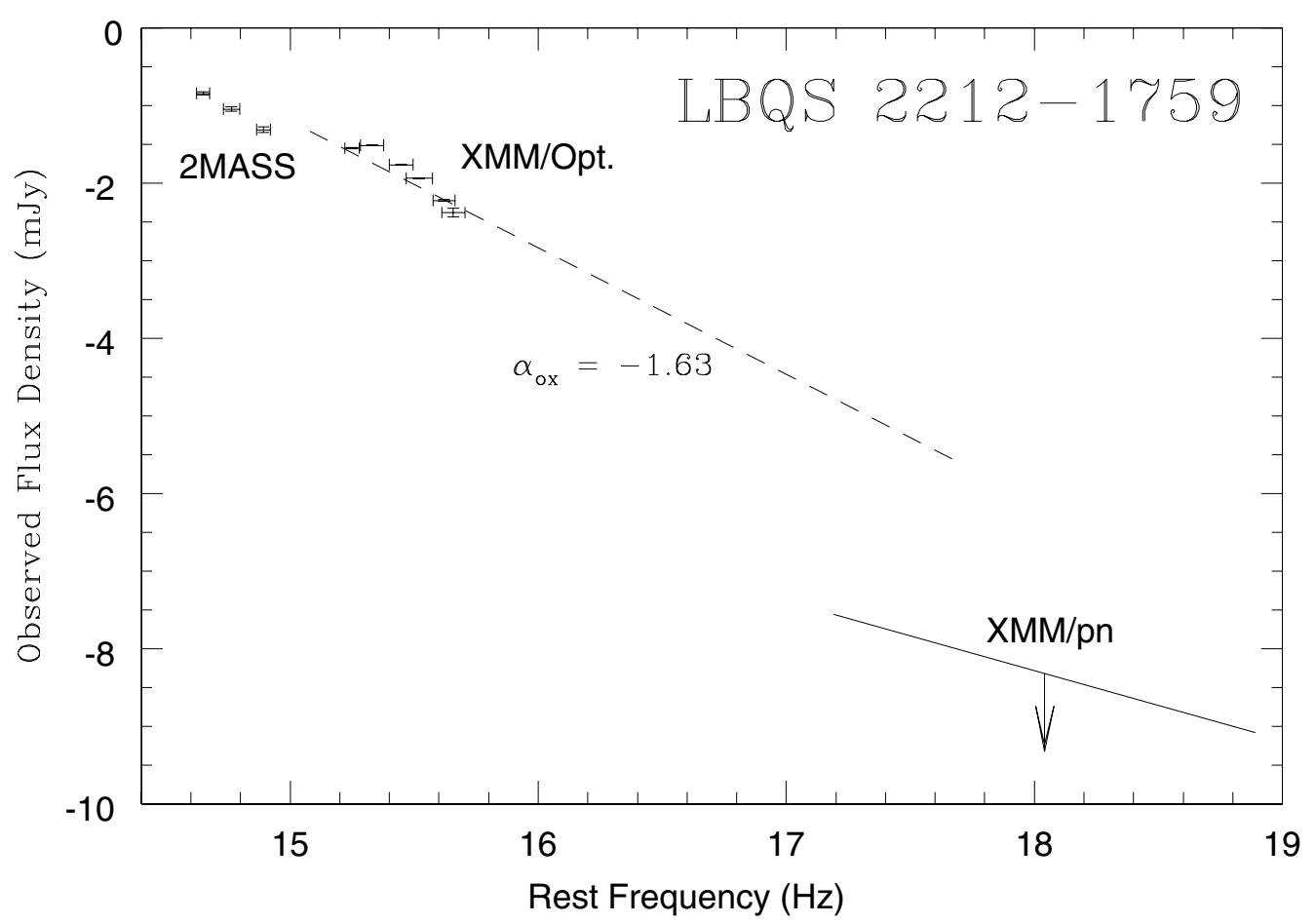

Fig. 2. The overall optical-to-X-ray spectral energy distribution of LBQS 2212-1759. All flux values are in the observer's frame and have been corrected for foreground galactic extinction $\left(E_{b-v}=0.026\right)$. The upper limit to the $0.2-10 \mathrm{keV}$ (observer's frame) flux of Table 2 is shown as a spectrum of photon index $\Gamma=-1.9$. For comparison, a fiducial power-law of index $\alpha_{\mathrm{ox}}=-1.63$ and extending from $2500 \AA$ to $2 \mathrm{keV}$ (rest frame) is shown as a dashed line. The LBQS 2212-1759 flux upper limit is 263 times lower than the flux predicted by extrapolating the $\alpha_{\mathrm{ox}}$ power-law to $2 \mathrm{keV}$.

in the UV and EUV range, and on the other it is not detected in the X-rays, with upper limits on the $0.2-10 \mathrm{keV}$ flux which, taken at face value, imply column densities sufficient to extinguish its ultraviolet emission as well.

In what follows, we briefly explore two possible explanations for this apparent contradiction:

1. LBQS 2212-1759 is genuinely $X$-ray weak, with an intrinsic optical to $X$-ray spectral index $\alpha_{\mathrm{ox}} \leq-2.56$ : available data, however, do not seem to support this hypothesis since the majority of BALQSOs, once corrected for absorption, have a normal energy distribution. For instance, the 8 BALQSOs observed by Gallagher et al. (2002) with Chandra have a mean optical to X-ray spectral index $\left\langle\alpha_{\text {ox }}\right\rangle=-1.58$, with an rms dispersion of 0.11. Similarly, the $6 \mathrm{HI}-\mathrm{BAL}$ quasars detected by Green et al. (2001) also with Chandra have a mean index $\left\langle\alpha_{\mathrm{ox}}\right\rangle=-1.71$, with an rms dispersion of 0.13 . Two additional HI-BAL QSOs observed with $X$ MM-Newton by Grupe et al. (2003) have intrinsic values of $\alpha_{\mathrm{ox}}$ of -1.50 and -1.48 , respectively. All these indices are entirely compatible with the value expected for radio-quiet non-BAL QSOs and much flatter than the upper limit in LBQS 2212-1759, $\alpha_{\text {ox }} \leq-2.56$. We note that the two LO-BAL quasars detected by Green et al. (2001), IRAS $07598+6508$ and FIRST J0840+3633 have apparent steep indices of -2.34 and -2.11 , respectively, because they likely suffer from additional absorption which cannot be corrected for given the low signal-to-noise ratio of their X-ray spectra. We note that LBQS 2212-1759 is definitely not a LO-BAL since its spectrum lacks characteristic absorption lines from low ionisation species, such as SiII $\lambda 1808$, AlIII $\lambda 1854$ and MgII $\lambda 2798$ (see Fig. 3). Only two BALQSOs seem to be genuinely X-ray deficient: with $\alpha_{\text {ox }}=-1.96$, PHL 1811 remains marginally X-ray weak after correction for internal absorption, though this conclusion is not very robust given the paucity of X-ray photons detected (183: Leighly et al. 2001); the second BALQSO is PG 1254+047 for which Sabra and Hamann (2001) present convincing evidence that it is at the same time intrinsically $\mathrm{X}$-ray weak $\left(\alpha_{\mathrm{ox}} \leq-2.0\right)$ and heavily absorbed $\left(N_{\mathrm{H}}=\right.$ $\left.2.8 \times 10^{23} \mathrm{~cm}^{2}\right)$. We note that the indices of both sources are still significantly larger than the upper limit on $\alpha_{\text {ox }}$ of LBQS 2212-1759. One can in principle gauge the slope of the optical-to-X-ray spectrum of LBQS 2212-1759 from its relative emission line intensities. Indeed, if the QSO was lacking EUV and soft X-rays, one would expect the high ionisation emission lines to be relatively weak compared to lines from lower ionisation species. In Table 5, we list the (de-reddened) intensities of the main emission lines together with their Full-Width at Half-Maximum (FWHM). The HeII $\lambda 1640$ line is undetected, which could be indicative of a deficiency in EUV and soft X-rays. However, the upper limit on its intensity relative to that of e.g. CIV $\lambda 1249$, 0.12 , is still higher than that in the average composite SDSS spectrum of Vanden Berk et al. (2001), 0.02, and is thus not particularly useful. Furthermore, the NV $\lambda 1240$ 

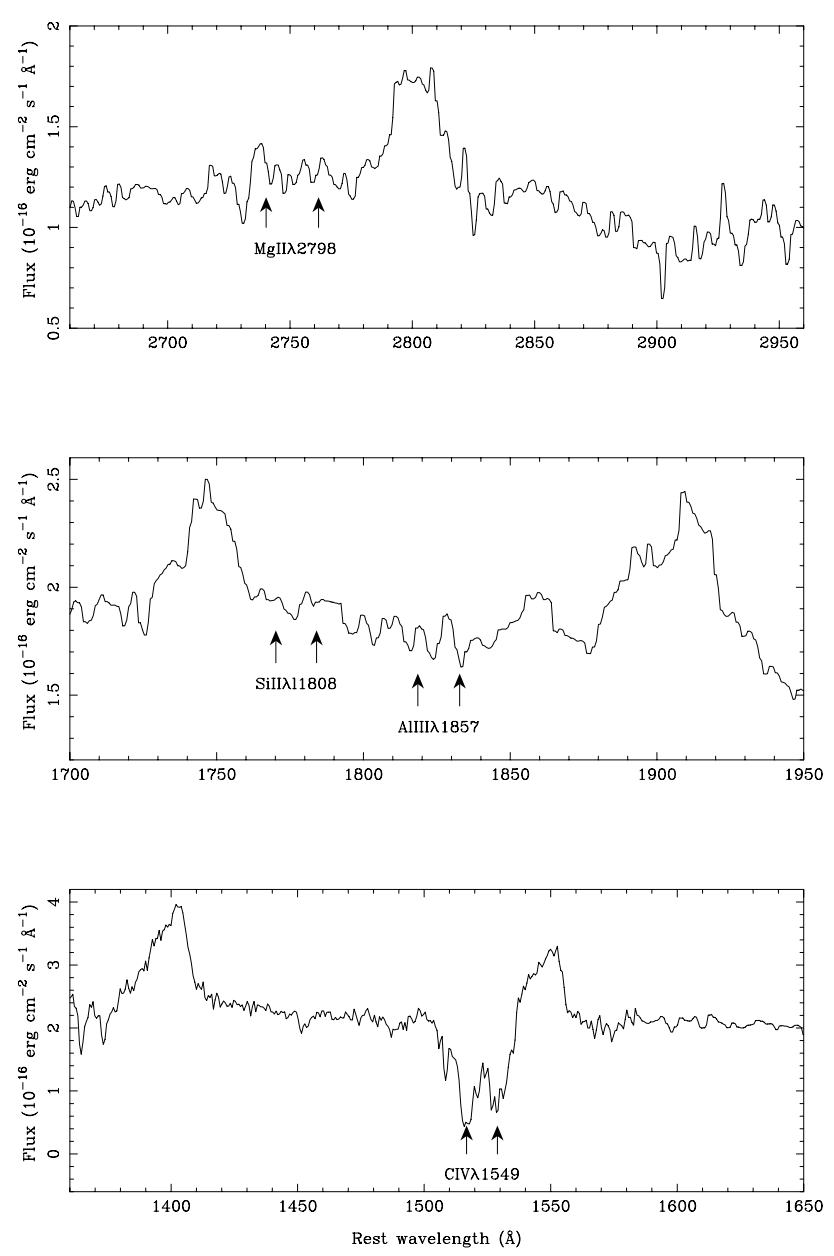

Fig. 3. The spectrum of LBQS 2212-1759 around the MgII $\lambda 2798$ line (upper panel), the AlIII $\lambda 1857$ and SiII $\lambda 1808$ lines (middle), and the CIV $\lambda 1549$ line (bottom). The wavelengths at which one expects blueshifted double absorption troughs are indicated with arrows. Note the absence of significant absorption from MgII, SiII and AlIII.

line is quite strong, with an intensity relative to $\operatorname{Ly} \alpha \lambda 1216$, $\mathrm{NV} / \mathrm{Ly} \alpha \simeq 0.65$, compared to e.g. 0.025 in the average composite SDSS spectrum. Since the photon energy required to ionize $\mathrm{N}^{3+}$ into $\mathrm{N}^{4+}$ is $77.5 \mathrm{eV}$, much larger than the $\mathrm{He}^{+}$ionisation potential, $24.6 \mathrm{eV}$, a deficit of EUV and soft $\mathrm{X}$-ray photons seems implausible. In fact, the NIII] $\lambda 1750$ is also quite strong (see also Fig. 3), with an intensity relative to $\operatorname{Ly} \alpha \lambda 1216, \mathrm{III}] / \operatorname{Ly} \alpha \simeq 0.12,32$ times larger than that in the average composite SDSS QSO spectrum (Vandeb Berk et al. 2001). This suggests an overabundance of Nitrogen in LBQS 2212-1759. The CIII] $\lambda 1909 /$ CIV $\lambda 1549$ intensity ratio, 1.49 , is 2.3 times larger than that of the composite SDSS QSO (0.63), but this is partly due to absorption eating away a fraction of the blue wing of the CIV emission line. In summary, the emission line intensity ratios do not provide conclusive evidence for a deficit in EUV and soft X-rays in LBQS 2212-1759.

2. The LOS to the optical-UV source is different from that to the X-ray source: with a "balnicity index" (Weyman et al. 1991) of $2221 \mathrm{~km} \mathrm{~s}^{-1}$ (Korista et al. 1993), LBQS 2212-1759 exhibits relatively weak BAL. Moreover,
Table 5. Intensities and full width at half maximum ( $F W H M)$ of the main emission lines in the spectrum of LBQS 2212-1759; all intensities are in units of $10^{-16} \mathrm{erg} \mathrm{cm}^{-2} \mathrm{~s}^{-1}$ and have been corrected for galactic extinction. Their uncertainties are typically $10 \%$. The FWHM are expressed in $\mathrm{km} \mathrm{s}^{-1}$ and have an uncertainty of $\pm 50 \mathrm{~km} \mathrm{~s}^{-1}$.

\begin{tabular}{lcc}
\hline \hline Line ID & Flux & $F W H M$ \\
\hline H-Ly $\alpha 1216$ & 204 & 1610 \\
NV $\lambda 1240$ & 132 & 4780 \\
SiIV $\lambda 1397$ & 50 & 3600 \\
CIV $\lambda 1549$ & 33 & 2790 \\
HeII $\lambda 1640$ & $\leq 4$ & - \\
NIII $\lambda \lambda 1750$ & 25 & 2780 \\
CIII $] \lambda 1909$ & 49 & 5150 \\
MgII $\lambda 2798$ & 41 & 2590 \\
\hline
\end{tabular}

only about $80 \%$ of its continuum flux is absorbed at the bottom of the CIV $\lambda 1550$ trough, indicating partial coverage of the UV source (Korista et al. 1993). Similarly, model-fits of the X-ray spectrum of the few BALQSOs detected at high energies also tend to require partial covering of the X-ray source (although models with an ionized absorber yield equally acceptable fits). In lower luminosity AGNs, different variability timescales in the two wavebands clearly demonstrate that the X-ray source is $\sim$ an order of magnitude more compact than the UV-optical source. It thus remains a possibility that, in some BALQSOs at least, the outflow intercepts only a fraction of the UV-optical emission while completely blocking-out the X-ray flux. Gallagher et al. (2004) reach a similar conclusion for the BALQSO PG $2112+059$ based on dramatically different patterns of variability in the X-ray and the UV regime. In PG $2112+059$ as well, the absence of a Lyman edge sets an upper limit to the UV continuum absorbing column $N_{\mathrm{H}} \leq 10^{17} \mathrm{~cm}^{-2}, 5-6$ orders of magnitude lower than the absorbing column covering the $\mathrm{X}$-ray source.

In the absence of a better choice, hypothesis number two remains our preferred explanation for the peculiar SED of LBQS 2212-1759, although we cannot rule-out the alternative possibility that LBQS $2212-1759$ is genuinely X-ray weak, with an intrinsically steep index, $\alpha_{\mathrm{ox}} \leq-2.56$. Obviously more observations with Chandra and XMM-Newton are required to increase the number of BALQSOs with measured X-ray properties or at least with stringent upper limits.

Acknowledgements. The authors are grateful to Kirk Korista for providing his 1992 optical spectrum of LBQS 2212-1759 in electronic form. The anonymous referee is also thanked for constructive comments which significantly improved this article.

This publication makes use of data products from the Two Micron All Sky Survey, which is a joint project of the University of Massachusetts and the Infrared Processing and Analysis Center/California Institute of Technology, funded by the National Aeronautics and Space Administration and the National Science Foundation.

This research has made use of the NASA/IPAC Extragalactic Database (NED) which is operated by the Jet Propulsion Laboratory, California Institute of Technology, under contract with the National Aeronautics and Space Administration. 


\section{References}

Arav, N., Korista, K. T., de Kool, M., Junkkarinen, V. T., \& Begelman, M. C. 1999, ApJ, 516, 27

Barkhouse, W. A., \& Hall, P. B. 2001, AJ, 121, 2843

Becker, H. R., White, R. L., Gregg, M. D., et al. 2000, ApJ, 538, 72

Boroson, T. A. 2002, ApJ, 565, 78

Briggs, F. H., Turnshek, D. A., \& Wolfe, A. M. 1984, ApJ, 287, 549

Cardelli, J. A., Clayton, G. C., \& Mathis, J. S. 1989, ApJ, 345, 245

den Herder, J. W., Brinkman, A. C., Kahn, S. M., et al. 2001, A\&A, $365, \mathrm{~L} 7$

Elvis, M., Wilkes, B. J., McDowell, J. C., et al. 1994. ApJS, 95, 1

Elvis, M. 2000, ApJ, 545, 63

Francis, P. J., Hewett, P. C., Foltz, C. B., et al. 1991, ApJ, 373, 465

Gallagher, S. C., Brandt, W. N., Sambruna, R. M., Mathur, S., \& Yamasaki, N. 1999, ApJ, 519, 549

Gallagher, S. C., Brandt, W. N., Chartas, G., \& Garmire, G. P. 2002, ApJ, 567, 37

Gallagher, S. C., Brandt, W. N., Wills, B. J., et al. 2004, ApJ, 603, 425

Gorenstein, P. 1975, ApJ, 198, 95

Green, P. J., Schartel, N., Anderson, S. F., et al. 1995, ApJ, 450, 51

Green, P. J, \& Mathur, S. 1996, ApJ, 462, 637

Green, P. J., Aldcroft, T. L., Mathur, S., Wilkes, B. J., \& Elvis, M. 2001, ApJ, 558, 109

Grupe, D., Mathur, S., \& Elvis, M. 2003, AJ, 126, 1159

Haardt, F., \& Maraschi, L. 1991, ApJ, 380, L51

Hewett, P. C., \& Foltz, C. B. 2003, AJ, 125, 1784

Jansen, F., Lumb, D., Altieri, B., et al. 2001, A\&A, 365, L1

Kleinmann, S. G., Lysaght, M. G., Pughe, W. L., et al. 1994, Ap\&SS, 217, 11

Korista, K. T., Voit, G. M., Morris, S. L., \& Weymann, R. J. 1993 , ApJS, 88,357

Kuhn, O., Elvis, M., Bechtold, J., \& Elston, R. 2001, ApJS, 136, 225
Laor, A., Fiore, F., Elvis, M., et al. 1997, ApJ, 477, 93

Leighly, K. M., Halpern, J. P., Helfand, D. J., Becker, R. H., \& Impey, C. D. 2001, AJ, 121, 2889

Mason, K. O., Breeveld, A., Much, R., et al. 2001, A\&A, 365, L36

Menou, K., Vanden Berk, D. E., Ivezic, Z., et al. 2001, ApJ, 561, 645

Monet, D., et al. 1998, USNO-A V2.0, A Catalog of Astrometric Standards U.S. Naval Observatory Flagstaff Station (USNOFS) and Universities Space Research Association (USRA) stationed at USNOFS

Morris, S. L., Weymann, R. J., Anderson, S. F., et al. 1991, AJ, 102, 1627

Murray, N., Chiang, J., Grossman, S. A., \& Voit, G. M. 1995, ApJ, 451, 498

Ogle, P. M., Cohen, M. H., Miller, J. S., et al. 1999, ApJS, 125, 1

Proga, D., Stone, J. M., \& Kallman, T. R. 2000, ApJ, 543, 686

Sabra, B. M., \& Hamann, F. 2001, ApJ, 563, 555

Schmidt, G. D., \& Hines, D. C. 1999, ApJ, 512, 125

Schlegel, D. J., Finkbeiner, D. P., \& Davis, M. 1998, ApJ, 500, 525

Strateva, I. V., Brandt, W. N., Schneider, D. P., Vanden Berk, D. G., \& Vignali, C. 2005, AJ, 130, 387

Strüder, L., Briel, U., Dennerl, K., et al. 2001, A\&A, 365, L18

Turner, M. J. L., Abbey, A., Arnaud, M., et al. 2001, A\&A, 365, L27

Vanden Berk, D. E., Richards, G. T., Bauer, A., et al. 2001, AJ, 122, 549

Vignali, C., Brandt, W. N., \& Schneider, D. P. 2003, AJ, 125, 433

Wang, T. G., Wang, J. X., Brinkmann, W., \& Matsuoka, M. 1999, ApJ, 519, L35

Weyman, R. J., Morris, S. L., Foltz, C. B., \& Hewett, P. C. 1991, ApJ, 373,23

Zamorani, G., Henry, J. P., Maccacaro, T., et al. 1981, ApJ, 245, 357

Zheng, W., Kriss, G. A., Telfer, R. C., Grimes, J. P., \& Davidsen, A. F. 1997, ApJ, 475, 469 